\title{
ON A SHARPER FORM OF HALF-DISCRETE HILBERT INEQUALITY
}

\author{
L. E. AZAR
}

Abstract. In this paper we introduce a general inequality by using the half-discrete Hilbert inequality. As an application we give a sharper form of the half-discrete Hilbert inequality and some carlson's type inequalities.

\section{Introduction}

If $f(x), g(x) \geq 0,0<\int_{0}^{\infty} f^{2}(x) d x<\infty$, and $0<\int_{0}^{\infty} g^{2}(x) d x<\infty$, then (see [3])

$$
\int_{0}^{\infty} \int_{0}^{\infty} \frac{f(x) g(y)}{x+y} d x d y<\pi\left\{\int_{0}^{\infty} f^{2}(x) d x\right\}^{\frac{1}{2}}\left\{\int_{0}^{\infty} g^{2}(x) d x\right\}^{\frac{1}{2}} .
$$

Inequality (1.1) is called Hilbert's integral inequality which has been extended by Hardy [3] as: if $p>1, \frac{1}{p}+\frac{1}{q}=1, f(x), g(x)>0,0<\int_{0}^{\infty} f^{p}(x) d x<\infty$, and $0<\int_{0}^{\infty} g^{q}(x) d x<\infty$, then

$$
\int_{0}^{\infty} \int_{0}^{\infty} \frac{f(x) g(y)}{x+y} d x d y<\frac{\pi}{\sin \frac{\pi}{p}}\left\{\int_{0}^{\infty} f^{p}(x) d x\right\}^{\frac{1}{p}}\left\{\int_{0}^{\infty} g^{q}(x) d x\right\}^{\frac{1}{q}} .
$$

The corresponding inequalities in the discrete case are $\left(a_{m}, b_{n}>0\right)$ :

$$
\begin{aligned}
& \sum_{n=1}^{\infty} \sum_{m=1}^{\infty} \frac{a_{m} b_{n}}{m+n}<\pi\left\{\sum_{n=1}^{\infty} a_{n}^{2}\right\}^{\frac{1}{2}}\left\{\sum_{n=1}^{\infty} b_{n}^{2}\right\}^{\frac{1}{2}} \\
& \sum_{n=1}^{\infty} \sum_{m=1}^{\infty} \frac{a_{m} b_{n}}{m+n}<\frac{\pi}{\sin \frac{\pi}{p}}\left\{\sum_{n=1}^{\infty} a_{n}^{p}\right\}^{\frac{1}{p}}\left\{\sum_{n=1}^{\infty} b_{n}^{q}\right\}^{\frac{1}{q}},
\end{aligned}
$$

provided that the series on the right-hand side of (1.3) and (1.4) are convergent. The constant factor $\pi$ is the best possible in both (1.1) and (1.3), and the constant $\frac{\pi}{\sin \frac{\pi}{p}}$ is the best possible in (1.2) and (1.4). Recently in [4] the following sharper forms of (1.2) and (1.4) were given

$$
\int_{0}^{\infty} \int_{0}^{\infty} \frac{f(x) g(y)}{x+y} d x d y<\sqrt{L}\left(\int_{0}^{\infty} x^{p q A_{1}-1} f^{p}(x) d x\right)^{\frac{1}{2 p}}\left(\int_{0}^{\infty} y^{p q A_{2}-1} g^{q}(y) d y\right)^{\frac{1}{2 q}}
$$

Received May 5, 2013, accepted September 13, 2013.

2010 Mathematics Subject Classification. 26D15.

Key words and phrases. Hilbert's inequality, Carlson's inequality, Cauchy's inequality, sharper form. 
and

$$
\times\left\{\int_{0}^{\infty} \int_{0}^{\infty} \frac{x f(x) g(y)}{(x+y)^{2}} d x d y\right\}^{\frac{p A_{2}}{2}}\left\{\int_{0}^{\infty} \int_{0}^{\infty} \frac{y f(x) g(y)}{(x+y)^{2}} d x d y\right\}^{\frac{q A_{1}}{2}}
$$

$$
\begin{aligned}
\sum_{n=1}^{\infty} \sum_{m=1}^{\infty} \frac{a_{m} b_{n}}{m+n}< & \sqrt{L}\left\{\sum_{m=1}^{\infty} m^{p q A_{1}-1} a_{m}^{p}\right\}^{\frac{1}{2 p}}\left\{\sum_{n=1}^{\infty} n^{p q A_{2}-1} b_{n}^{q}\right\}^{\frac{1}{2 q}} \\
& \times\left\{\sum_{n=1}^{\infty} \sum_{m=1}^{\infty} \frac{n a_{m} b_{n}}{(m+n)^{2}}\right\}^{\frac{q A_{1}}{2}}\left\{\sum_{n=1}^{\infty} \sum_{m=1}^{\infty} \frac{m a_{m} b_{n}}{(m+n)^{2}}\right\}^{\frac{p A_{2}}{2}},
\end{aligned}
$$

here $A_{1} \in\left(0, \frac{1}{q}\right), A_{2} \in\left(0, \frac{1}{p}\right), p A_{2}+q A_{1}=1$ and the constant $L=\frac{B\left(p A_{2}, 1-p A_{2}\right)}{\left(p A_{2}\right)^{p A_{2}}\left(q A_{1}\right)^{q A_{1}}}(B(x, y)$ is the Beta function). Note that both (1.5) and (1.6) reduces to (1.2) and (1.4) if we put $A_{1}=A_{2}=\frac{1}{p q}$ and apply Young's Inequality, see [4].

In [1] Yang introduced the following half-discrete Hilbert's inequality

$$
\int_{0}^{\infty} f(x) \sum_{n=1}^{\infty} \frac{a_{n}}{(x+n)^{\lambda}} d x<B\left(\lambda_{1}, \lambda_{2}\right)\left(\int_{0}^{\infty} x^{p\left(1-\lambda_{1}\right)-1} f^{p}(x) d x\right)^{\frac{1}{p}}\left(\sum_{n=1}^{\infty} n^{q\left(1-\lambda_{2}\right)-1} a_{n}^{q}\right)^{\frac{1}{q}},
$$

here, $\lambda_{1}, \lambda_{2}>0, \lambda_{1}+\lambda_{2}=\lambda, 0<\lambda_{1}<1$, and the constant $B\left(\lambda_{1}, \lambda_{2}\right)$ is the best possible. In [2] B. Yang and Q. Chen obtained a general half-discrete Hilbert's inequality for a homogeneous kernel. In fact they proved the following theorem:

Theorem A. Suppose that $\lambda_{1}, \lambda_{2} \in \mathbb{R}, \lambda_{1}+\lambda_{2}=\lambda, k_{\lambda}(u(x), v(y))$ is a non-negative finite homogeneous function of degree $-\lambda$ in $\mathbb{R}_{+s}^{2} u(x)(x \in(b, c),-\infty \leq b<c \leq \infty)$ and $v(y)\left(y \in\left[n_{0}, \infty\right), n_{0} \in\right.$ $\mathbb{N})$ are strictly increasing differential functions with $u\left(b^{+}\right)=0, v\left(n_{0}\right)>0, u\left(c^{-}\right)=v(\infty)=\infty$, $k\left(\lambda_{1}\right) \in \mathbb{R}_{+}$. If $p>1, \frac{1}{p}+\frac{1}{q}=1, f(x), a_{n} \geq 0, \int_{b}^{c} \frac{[u(x)]^{p\left(1-\lambda_{1}\right)-1}}{\left[u^{\prime}(x)\right]^{p-1}} f^{p}(x) d x<\infty, 0<\sum_{n=n_{0}}^{\infty} \frac{[v(n)]^{q\left(1-\lambda_{2}\right)-1}}{\left[v^{\prime}(n)\right]^{q-1}} a_{n}^{q}$ $<\infty$ then we have the following inequality:

$$
\begin{aligned}
& \sum_{n=n_{0}}^{\infty} a_{n} \int_{b}^{c} k_{\lambda}(u(x), v(n)) f(x) d x=\int_{b}^{c} f(x) \sum_{n=n_{0}}^{\infty} a_{n} k_{\lambda}(u(x), v(n)) d x \\
& \quad<k\left(\lambda_{1}\right)\left(\int_{b}^{c} \frac{[u(x)]^{p\left(1-\lambda_{1}\right)-1}}{\left[u^{\prime}(x)\right]^{p-1}} f^{p}(x) d x\right)^{\frac{1}{p}}\left(\sum_{n=n_{0}}^{\infty} \frac{[v(n)]^{q\left(1-\lambda_{2}\right)-1}}{\left[v^{\prime}(n)\right]^{q-1}} a_{n}^{q}\right)^{\frac{1}{q}} .
\end{aligned}
$$

Moreover, if $\frac{v^{\prime}(y)}{v(y)}\left(y \geq n_{0}\right)$ is decreasing and there exist constants $\mathbf{\square}<\lambda_{1}$ and $M>0$, such that $k_{\lambda}(t, 1) \leq \frac{M}{t^{\delta}} t \in\left(0, \frac{1}{v\left(n_{0}\right)}\right)$, then the constant factor $k\left(\lambda_{1}\right)$ is the best possible.

In particular, if we put $k_{1}(u(x), v(n))=\frac{1}{\alpha u(x)+\beta v(n)}, \lambda_{1}+\lambda_{2}=1(\alpha, \beta>0)$, we get from (1.8)

$$
\sum_{n=n_{0}}^{\infty} a_{n} \int_{b}^{c} \frac{1}{\alpha u(x)+\beta v(n)} f(x) d x<\frac{B\left(\lambda_{1}, \lambda_{2}\right)}{\alpha^{\lambda_{1}} \beta^{\lambda_{2}}}\left(\int_{b}^{c} \frac{[u(x)]^{p\left(1-\lambda_{1}\right)-1}}{\left[u^{\prime}(x)\right]^{1-1}} f^{p}(x) d x\right)^{\frac{1}{p}}\left(\sum_{n=n_{0}}^{\infty} \frac{[\nu(n)]^{q\left(1-\lambda_{2}\right)-1}}{\left[\nu^{\prime}(n)\right]^{q-1}} a_{n}^{q}\right)^{\frac{1}{q}} .
$$


We need the following formula for the beta function

$$
B(s, t+1)=\frac{t}{s+t} B(s, t) .
$$

For the sequence of real numbers $\left(a_{n}\right)$, Carlson's inequality is given as

$$
\sum_{n=1}^{\infty} a_{n}<\sqrt{\pi}\left(\sum_{n=1}^{\infty} a_{n}^{2}\right)^{\frac{1}{4}}\left(\sum_{n=1}^{\infty} n^{2} a_{n}^{2}\right)^{\frac{1}{4}}
$$

the constant $\sqrt{\pi}$ is the best possible. The continuous version of (1.10) is

$$
\int_{0}^{\infty} f(x) d x \leq \sqrt{\pi}\left(\int_{0}^{\infty} f^{2}(x) d x\right)^{\frac{1}{4}}\left(\int_{0}^{\infty} x^{2} f^{2}(x) d x\right)^{\frac{1}{4}},
$$

the constant $\sqrt{\pi}$ is sharp. For more details about these inequalities and their extensions we refer the reader to the book [5].

In this paper, we introduce a new inequality with a best constant factor which gives an upper estimate for the quantity $\sum_{n=n_{0}}^{\infty} \int_{b}^{c} F(x, n) d x$, where $F(x, n)$ is a positive function defined on $(b, c) \times\left(n_{0}, \infty\right)$. As an application, we obtain a sharper form of half-discrete Hilbert inequality (1.9). Some examples of Carlson's type inequalities are also considered.

\section{Main results}

Theorem 2.1. Let $p>1, \frac{1}{p}+\frac{1}{q}=1$, the functions $u, v, f$ and $a_{n}$ are as in Theorem $A, 0<$ $\int_{b}^{c} \frac{[u(x)]^{p\left(1-\lambda_{1}\right)-1}}{\left[u^{\prime}(x)\right]^{p-1}} f^{p}(x) d x<\infty, 0<\sum_{n=n_{0}}^{\infty} \frac{[v(n)]^{q\left(1-\lambda_{2}\right)-1}}{\left[\nu^{\prime}(n)\right]^{q-1}} a_{n}^{q}<\infty$. Let $F(x, n)$ be a positive function defined on $(b, c) \times\left(n_{0}, \infty\right)$ such that $\sum_{n=n_{0}}^{\infty} \int_{b}^{c} \frac{u(x) F^{2}(x, n)}{a_{n} f(x)} d x<\infty$ and $\sum_{n=n_{0}}^{\infty} \int_{b}^{c} \frac{v(n) F^{2}(x, n)}{a_{n} f(x)} d x<\infty$ then the following inequality holds

$$
\begin{aligned}
{\left[\sum_{n=n_{0}}^{\infty} \int_{b}^{c} F(x, n) d x\right]^{2}<} & K\left(\int_{b}^{c} \frac{[u(x)]^{p\left(1-\lambda_{1}\right)-1}}{\left[u^{\prime}(x)\right]^{p-1}} f^{p}(x) d x\right)^{\frac{1}{p}}\left(\sum_{n=n_{0}}^{\infty} \frac{[v(n)]^{q\left(1-\lambda_{2}\right)-1}}{\left[v^{\prime}(n)\right]^{q-1}} a_{n}^{q}\right)^{\frac{1}{q}} \\
& \times\left(\sum_{n=n_{0}}^{\infty} \int_{b}^{c} \frac{u(x) F^{2}(x, n)}{a_{n} f(x)} d x\right)^{\lambda_{1}}\left(\sum_{n=n_{0}}^{\infty} \int_{b}^{c} \frac{v(n) F^{2}(x, n)}{a_{n} f(x)} d x\right)^{\lambda_{2}},
\end{aligned}
$$

here $\lambda_{1}, \lambda_{2}>0, \lambda_{1}+\lambda_{2}=1$ and the constant $K=\frac{B\left(\lambda_{1}, \lambda_{2}\right)}{\lambda_{1}^{\lambda_{1}} \lambda_{2}^{\lambda_{2}}}$ is the best possible.

Proof. Let $\alpha, \beta>0$, applying Cauchy's inequality for integrals, Cauchy's inequality for series and inequality (1.9), respectively, we get

$$
\left[\sum_{n=n_{0}}^{\infty} \int_{b}^{c} F(x, n) d x\right]^{2}
$$




$$
\begin{aligned}
& =\left[\sum_{n=n_{0}}^{\infty} \int_{b}^{c}\left\{\frac{\sqrt{a_{n} f(x)}}{\sqrt{\alpha u(x)+\beta v(n)}}\right\}\left\{\frac{\sqrt{\alpha u(x)+\beta v(n)}}{\sqrt{a_{n} f(x)}} F(x, n)\right\} d x\right]^{2} \\
& \leq\left[\sum_{n=n_{0}}^{\infty}\left(\int_{b}^{c} \frac{a_{n} f(x) d x}{\alpha u(x)+\beta v(n)}\right)^{\frac{1}{2}}\left(\int_{b}^{c} \frac{\alpha u(x)+\beta v(n)}{a_{n} f(x)} F^{2}(x, n) d x\right)^{\frac{1}{2}}\right]^{2} \\
& \leq \sum_{n=n_{0}}^{\infty} \int_{b}^{c} \frac{a_{n} f(x) d x}{\alpha u(x)+\beta v(n)} \sum_{n=n_{0}}^{\infty} \int_{b}^{c} \frac{\alpha u(x)+\beta v(n)}{a_{n} f(x)} F^{2}(x, n) d x \\
& <\frac{B\left(\lambda_{1}, \lambda_{2}\right)}{\alpha^{\lambda_{1}} \beta^{\lambda_{2}}}\left(\int_{b}^{c} \frac{[u(x)]^{p\left(1-\lambda_{1}\right)-1}}{\left[u^{\prime}(x)\right]^{p-1}} f^{p}(x) d x\right)^{\frac{1}{p}}\left(\sum_{n=n_{0}}^{\infty} \frac{[v(n)]^{q\left(1-\lambda_{2}\right)-1}}{\left[v^{\prime}(n)\right]^{q-1}} a_{n}^{q}\right)^{\frac{1}{q}} \\
& \times\left[\alpha \sum_{n=n_{0}}^{\infty} \int_{b}^{c} \frac{u(x)}{a_{n} f(x)} F^{2}(x, n) d x+\beta \sum_{n=n_{0}}^{\infty} \int_{b}^{c} \frac{v(n)}{a_{n} f(x)} F^{2}(x, n) d x\right] \\
& =B\left(\lambda_{1}, \lambda_{2}\right)\left(\int_{b}^{c} \frac{[u(x)]^{p\left(1-\lambda_{1}\right)-1}}{\left[u^{\prime}(x)\right]^{p-1}} f^{p}(x) d x\right)^{\frac{1}{p}}\left(\sum_{n=n_{0}}^{\infty} \frac{[v(n)]^{q\left(1-\lambda_{2}\right)-1}}{\left[v^{\prime}(n)\right]^{q-1}} a_{n}^{q}\right)^{\frac{1}{q}} \\
& \times\left[\left(\frac{\alpha}{\beta}\right)^{\lambda_{2}} \sum_{n=n_{0}}^{\infty} \int_{b}^{c} \frac{u(x)}{a_{n} f(x)} F^{2}(x, n) d x+\left(\frac{\beta}{\alpha}\right)^{\lambda_{1}} \sum_{n=n_{0}}^{\infty} \int_{b}^{c} \frac{v(n)}{a_{n} f(x)} F^{2}(x, n) d x\right] .
\end{aligned}
$$

Set $S=\sum_{n=n_{0}}^{\infty} \int_{b}^{c} \frac{u(x)}{a_{n} f(x)} F^{2}(x, n) d x, T=\sum_{n=n_{0}}^{\infty} \int_{b}^{c} \frac{v(n)}{a_{n} f(x)} F^{2}(x, n) d x, y=\frac{\beta}{\alpha}$ and consider the function $g(y)=y^{-\lambda_{2}} S+y^{\lambda_{1}} T$. Since $g^{\prime}(y)=y^{\lambda_{1}-2}\left(\lambda_{1} T y-\lambda_{2} S\right)$, we conclude that the minimum of this function attains for $y=\frac{\lambda_{2} S}{\lambda_{1} T}$. Therefore, if we let $\alpha=\lambda_{1} T$ and $\beta=\lambda_{2} S$, we get (2.1).

It remains to show that the constant $K$ in (2.1) is the best possible. There exists $d \in(b, c)$, satisfying $u(d)=1$. For $0<\varepsilon<p \lambda_{1}$, setting $\widetilde{f}(x)=0, x \in(b, d) ; \widetilde{f}(x)=[u(x)]^{\lambda_{1}-\frac{\varepsilon}{p}-1} u^{\prime}(x), x \in$ $[d, c), \widetilde{a}_{n}=[v(n)]^{\lambda_{2}-\frac{\varepsilon}{q}-1} v^{\prime}(n), n \geq n_{0}$ and $\widetilde{F}(x, n)=\frac{\widetilde{a}_{n} \widetilde{f}(x)}{u(x)+v(n)}$. Suppose that there exists a positive constant $C<K$ such that (2.1) is still valid if we replace $K$ by $C$, then we find

$$
\begin{aligned}
\widetilde{I}= & :\left[\sum_{n=n_{0}}^{\infty} \int_{b}^{c} \widetilde{F}(x, n) d x\right]^{2}=\left[\sum_{n=n_{0}}^{\infty} \int_{b}^{c} \frac{\widetilde{a}_{n} \widetilde{f}(x)}{u(x)+v(n)} d x\right]^{2} \\
< & C\left(\int_{b}^{c} \frac{[u(x)]^{p\left(1-\lambda_{1}\right)-1}}{\left[u^{\prime}(x)\right]^{p-1}} \widetilde{f}^{p}(x) d x\right)^{\frac{1}{p}}\left(\sum_{n=n_{0}}^{\infty} \frac{[v(n)]^{q\left(1-\lambda_{2}\right)-1}}{\left[v^{\prime}(n)\right]^{q-1}} \widetilde{a}_{n}^{q}\right)^{\frac{1}{q}} \\
& \times\left(\sum_{n=n_{0}}^{\infty} \int_{b}^{c} \frac{u(x) \widetilde{a}_{n} \widetilde{f}(x)}{(u(x)+v(n))^{2}} d x\right)^{\lambda_{1}}\left(\sum_{n=n_{0}}^{\infty} \int_{b}^{c} \frac{v(n) \widetilde{a}_{n} \tilde{f}(x)}{(u(x)+v(n))^{2}} d x\right)^{\lambda_{2}} \\
:= & C\left(I_{1}^{\frac{1}{p}}+I_{2}^{\frac{1}{q}}+I_{3}^{\lambda_{1}}+I_{4}^{\lambda_{2}}\right) .
\end{aligned}
$$

Computing these quantities we obtain

$$
I_{1}=\int_{d}^{c}[u(x)]^{-\varepsilon-1} u^{\prime}(x) d x=\int_{1}^{\infty} t^{-1-\varepsilon} d t=\frac{1}{\varepsilon}
$$




$$
\begin{aligned}
I_{2} & =\sum_{n=n_{0}}^{\infty}[v(n)]^{-\varepsilon-1} v^{\prime}(n)<\frac{v^{\prime}\left(n_{0}\right)}{\left[v\left(n_{0}\right)\right]^{1+\varepsilon}}+\int_{n_{0}}^{\infty}[v(t)]^{-\varepsilon-1} v^{\prime}(t) d t=\frac{v^{\prime}\left(n_{0}\right)}{\left[v\left(n_{0}\right)\right]^{1+\varepsilon}}+\frac{1}{\varepsilon\left[v\left(n_{0}\right)\right]^{\varepsilon}}, \\
I_{3} & =\sum_{n=n_{0}}^{\infty}[v(n)]^{\lambda_{2}-\frac{\varepsilon}{q}-1} v^{\prime}(n) \int_{d}^{c} \frac{[u(x)]^{\lambda_{1}-\frac{\varepsilon}{p}} u^{\prime}(x)}{(u(x)+v(n))^{2}} d x \\
& =\left(t=\frac{u(x)}{v(n)}\right)=\sum_{n=n_{0}}^{\infty}[v(n)]^{-\varepsilon-1} v^{\prime}(n) \int_{\frac{1}{v(n)}}^{\infty} \frac{t^{\lambda_{1}-\frac{\varepsilon}{p}}}{(t+1)^{2}} d x \\
& <\sum_{n=n_{0}}^{\infty}[v(n)]^{-\varepsilon-1} v^{\prime}(n) \int_{0}^{\infty} \frac{t^{\lambda_{1}-\frac{\varepsilon}{p}}}{(t+1)^{2}} d x=\left[B\left(\lambda_{1}+1, \lambda_{2}\right)+o(1)\right] \sum_{n=n_{0}}^{\infty} \frac{v^{\prime}(n)}{[v(n)]^{\varepsilon+1}} \\
& <\left[B\left(\lambda_{1}+1, \lambda_{2}\right)+o(1)\right]\left(\frac{v^{\prime}\left(n_{0}\right)}{\left[v\left(n_{0}\right)\right]^{\varepsilon+1}}+\frac{1}{\varepsilon\left[v\left(n_{0}\right)\right]^{\varepsilon}}\right) .
\end{aligned}
$$

Similarly we obtain

$$
I_{4}<\left[B\left(\lambda_{1}, \lambda_{2}+1\right)+o(1)\right]\left(\frac{v^{\prime}\left(n_{0}\right)}{\left[v\left(n_{0}\right)\right]^{\varepsilon+1}}+\frac{1}{\varepsilon\left[v\left(n_{0}\right)\right]^{\varepsilon}}\right) .
$$

Now

$$
\begin{aligned}
& \sqrt{\widetilde{I}}=\sum_{n=n_{0}}^{\infty} \int_{b}^{c} \widetilde{F}(x, n) d x=\sum_{n=n_{0}}^{\infty} \int_{b}^{c} \frac{\widetilde{a}_{n} \widetilde{f}(x)}{u(x)+v(n)} d x \\
& =\sum_{n=n_{0}}^{\infty} \int_{d}^{c} \frac{[u(x)]^{\lambda_{1}-\frac{\varepsilon}{p}-1} u^{\prime}(x)[v(n)]^{\lambda_{2}-\frac{\varepsilon}{q}-1} v^{\prime}(n)}{u(x)+v(n)} d x=\left(t=\frac{u(x)}{v(n)}\right) \\
& =\sum_{n=n_{0}}^{\infty}[v(n)]^{-\varepsilon-1} v^{\prime}(n) \int_{\frac{1}{v(n)}}^{\infty} \frac{t^{\lambda_{1}-\frac{\varepsilon}{p}-1}}{t+1} d t \\
& =\sum_{n=n_{0}}^{\infty}[v(n)]^{-\varepsilon-1} v^{\prime}(n)\left(\int_{0}^{\infty} \frac{t^{\lambda_{1}-\frac{\varepsilon}{p}-1}}{t+1} d t-\int_{0}^{\frac{1}{v(n)}} \frac{t^{\lambda_{1}-\frac{\varepsilon}{p}-1}}{t+1} d t\right) \\
& =\left[B\left(\lambda_{1}, \lambda_{2}\right)+o(1)\right] \sum_{n=n_{0}}^{\infty}[v(n)]^{-\varepsilon-1} v^{\prime}(n)-\sum_{n=n_{0}}^{\infty}[v(n)]^{-\varepsilon-1} v^{\prime}(n) \int_{0}^{\frac{1}{\nu(n)}} \frac{t^{\lambda_{1}-\frac{\varepsilon}{p}-1}}{t+1} d t \\
& >\left[B\left(\lambda_{1}, \lambda_{2}\right)+o(1)\right]\left(\frac{v^{\prime}\left(n_{0}\right)}{\left[v\left(n_{0}\right)\right]^{\varepsilon+1}}+\frac{1}{\varepsilon\left[v\left(n_{0}\right)\right]^{\varepsilon}}\right)-\sum_{n=n_{0}}^{\infty}[v(n)]^{-\varepsilon-1} v^{\prime}(n) \int_{0}^{\frac{1}{\nu(n)}} t^{\lambda_{1}-\frac{\varepsilon}{p}-1} d t \\
& =\left[B\left(\lambda_{1}, \lambda_{2}\right)+o(1)\right]\left(\frac{v^{\prime}\left(n_{0}\right)}{\left[\nu\left(n_{0}\right)\right]^{\varepsilon+1}}+\frac{1}{\varepsilon\left[v\left(n_{0}\right)\right]^{\varepsilon}}\right)-\sum_{n=n_{0}}^{\infty} \frac{[v(n)]^{-\frac{\varepsilon}{q}-\lambda_{1}-1} v^{\prime}(n)}{\lambda_{1}-\frac{\varepsilon}{p}} \\
& >\left[B\left(\lambda_{1}, \lambda_{2}\right)+o(1)\right]\left(\frac{v^{\prime}\left(n_{0}\right)}{\left[v\left(n_{0}\right)\right]^{\varepsilon+1}}+\frac{1}{\varepsilon\left[\nu\left(n_{0}\right)\right]^{\varepsilon}}\right)-\frac{\left[v\left(n_{0}\right)\right]^{-\frac{\varepsilon}{q}-\lambda_{1}-1} \nu^{\prime}\left(n_{0}\right)}{\lambda_{1}-\frac{\varepsilon}{p}} \\
& -\int_{n_{0}}^{\infty} \frac{[\nu(n)]^{-\frac{\varepsilon}{q}-\lambda_{1}-1} v^{\prime}(n)}{\lambda_{1}-\frac{\varepsilon}{p}} \\
& =\left[B\left(\lambda_{1}, \lambda_{2}\right)+o(1)\right]\left(\frac{v^{\prime}\left(n_{0}\right)}{\left[v\left(n_{0}\right)\right]^{\varepsilon+1}}+\frac{1}{\varepsilon\left[v\left(n_{0}\right)\right]^{\varepsilon}}\right)-O(1) .
\end{aligned}
$$


Substituting the above inequalities in (2.1) we get

$$
\begin{aligned}
&\{[\left.\left.B\left(\lambda_{1}, \lambda_{2}\right)+o(1)\right]\left(\frac{v^{\prime}\left(n_{0}\right)}{\left[v\left(n_{0}\right)\right]^{\varepsilon+1}}+\frac{1}{\varepsilon\left[v\left(n_{0}\right)\right]^{\varepsilon}}\right)-O(1)\right\}^{2} \\
&< C\left(\frac{1}{\varepsilon}\right)^{\frac{1}{p}}\left(\frac{v^{\prime}\left(n_{0}\right)}{\left[v\left(n_{0}\right)\right]^{1+\varepsilon}}+\frac{1}{\varepsilon\left[v\left(n_{0}\right)\right]^{\varepsilon}}\right)^{\frac{1}{q}}\left(B\left(\lambda_{1}+1, \lambda_{2}\right)+o(1)\right)^{\lambda_{1}}\left(\frac{v^{\prime}\left(n_{0}\right)}{\left[v\left(n_{0}\right)\right]^{\varepsilon+1}}+\frac{1}{\varepsilon\left[v\left(n_{0}\right)\right]^{\varepsilon}}\right)^{\lambda_{1}} \\
& \quad \times\left(B\left(\lambda_{1}, \lambda_{2}+1\right)+o(1)\right)^{\lambda_{2}}\left(\frac{v^{\prime}\left(n_{0}\right)}{\left[v\left(n_{0}\right)\right]^{\varepsilon+1}}+\frac{1}{\varepsilon\left[v\left(n_{0}\right)\right]^{\varepsilon}}\right)^{\lambda_{2}} .
\end{aligned}
$$

Multiplying inequality (2.3) by $\varepsilon^{2}\left(\varepsilon=\varepsilon^{\lambda_{1}} \varepsilon^{\lambda_{2}}\right)$ and then let $\varepsilon \rightarrow 0^{+}$, we have

$$
B^{2}\left(\lambda_{1}, \lambda_{2}\right) \leq C B^{\lambda_{1}}\left(\lambda_{1}+1, \lambda_{2}\right) B^{\lambda_{2}}\left(\lambda_{1}, \lambda_{2}+1\right) .
$$

Using (1.10) we find

$$
B^{\lambda_{1}}\left(\lambda_{1}+1, \lambda_{2}\right)=\lambda_{1}^{\lambda_{1}} B^{\lambda_{1}}\left(\lambda_{1}, \lambda_{2}\right),
$$

and

$$
B^{\lambda_{2}}\left(\lambda_{1}, \lambda_{2}+1\right)=\lambda_{2}^{\lambda_{2}} B^{\lambda_{2}}\left(\lambda_{1}, \lambda_{2}\right)
$$

Substituting (2.5) and (2.6) in (2.4) we obtain the contradiction $C \geq K=\frac{B\left(\lambda_{1}, \lambda_{2}\right)}{\lambda_{1}^{\lambda_{1}} \lambda_{2}^{\lambda_{2}}}$. The Theorem is proved.

\section{Some Applications}

1. If we put $F(x, n)=\frac{a_{n} f(x)}{u(x)+v(n)}$ in (2.1), then we have the following form of half-discrete Hilbert's inequality

$$
\begin{aligned}
\left(\sum_{n=n_{0}}^{\infty} \int_{b}^{c} \frac{a_{n} f(x)}{u(x)+v(n)} d x\right)^{2}< & \frac{B\left(\lambda_{1}, \lambda_{2}\right)}{\lambda_{1}^{\lambda_{1}} \lambda_{2}^{\lambda_{2}}}\left(\int_{b}^{c} \frac{[u(x)]^{p\left(1-\lambda_{1}\right)-1}}{\left[u^{\prime}(x)\right]^{p-1}} f^{p}(x) d x\right)^{\frac{1}{p}}\left(\sum_{n=n_{0}}^{\infty} \frac{[v(n)]^{q\left(1-\lambda_{2}\right)-1}}{\left[v^{\prime}(n)\right]^{q-1}} a_{n}^{q}\right)^{\frac{1}{q}} \\
& \times\left(\sum_{n=n_{0}}^{\infty} \int_{b}^{c} \frac{u(x) f(x) a_{n}}{(u(x)+v(n))^{2}} d x\right)^{\lambda_{1}}\left(\sum_{n=n_{0}}^{\infty} \int_{b}^{c} \frac{v(n) f(x) a_{n}}{(u(x)+v(n))^{2}} d x\right)^{\lambda_{2}},(2.7)
\end{aligned}
$$

Inequality (2.7) is a sharper form of (1.9). To see that, let us rewrite (2.7) in the following form

$$
\left(\sum_{n=n_{0}}^{\infty} \int_{b}^{c} \frac{a_{n} f(x)}{u(x)+v(n)} d x\right)^{2}<\frac{B\left(\lambda_{1}, \lambda_{2}\right)}{\lambda_{1}^{\lambda_{1}} \lambda_{2}^{\lambda_{2}}}\left(\int_{b}^{c} \frac{[u(x)]^{p\left(1-\lambda_{1}\right)-1}}{\left[u^{\prime}(x)\right]^{p-1}} f^{p}(x) d x\right)^{\frac{1}{p}}\left(\sum_{n=n_{0}}^{\infty} \frac{[\nu(n)]^{q\left(1-\lambda_{2}\right)-1}}{\left[\nu^{\prime}(n)\right]^{q-1}} a_{n}^{q}\right)^{\frac{1}{q}} S^{\lambda_{1}} T^{\lambda_{2}},
$$

where $S=\sum_{n=n_{0}}^{\infty} \int_{b}^{c} \frac{u(x) f(x) a_{n}}{(u(x)+v(n))^{2}} d x$ and $T=\sum_{n=n_{0}}^{\infty} \int_{b}^{c} \frac{v(n) f(x) a_{n}}{(u(x)+v(n))^{2}} d x$. Since we may write $\sum_{n=n_{0}}^{\infty}$ $\int_{b}^{c} \frac{a_{n} f(x)}{u(x)+\nu(n)} d x=S+T$, dividing both sides of the last inequality by $\sum_{n=n_{0}}^{\infty} \int_{b}^{c} \frac{a_{n} f(x)}{u(x)+\nu(n)} d x$, we get 


$$
\begin{aligned}
\sum_{n=n_{0}}^{\infty} \int_{b}^{c} \frac{a_{n} f(x)}{u(x)+v(n)} d x< & B\left(\lambda_{1}, \lambda_{2}\right)\left(\int_{b}^{c} \frac{[u(x)]^{p\left(1-\lambda_{1}\right)-1}}{\left[u^{\prime}(x)\right]^{p-1}} f^{p}(x) d x\right)^{\frac{1}{p}}\left(\sum_{n=n_{0}}^{\infty} \frac{[v(n)]^{q\left(1-\lambda_{2}\right)-1}}{\left[v^{\prime}(n)\right]^{q-1}} a_{n}^{q}\right)^{\frac{1}{q}} \\
& \times \frac{\left(\frac{S}{\lambda_{1}}\right)^{\lambda_{1}}\left(\frac{T}{\lambda_{2}}\right)^{\lambda_{2}}}{S+T} .
\end{aligned}
$$

Applying Young's inequality ( $x^{\theta} y^{\mu} \leq \theta x+\mu y, \theta+\mu=1$ ) to the product $\left(\frac{S}{\lambda_{1}}\right)^{\lambda_{1}}\left(\frac{T}{\lambda_{2}}\right)^{\lambda_{2}}$ with $x=\frac{S}{\lambda_{1}}$ and $y=\frac{T}{\lambda_{2}}$, we obtain

$$
\frac{\left(\frac{S}{\lambda_{1}}\right)^{\lambda_{1}}\left(\frac{T}{\lambda_{2}}\right)^{\lambda_{2}}}{T+S} \leq 1
$$

Therefore, inequality (2.7) is a sharper form of (1.9). In particular if we set $u(x)=x, x \in(0, \infty)$ and $v(n)=n, n \geq 1$ we obtain

$$
\begin{aligned}
\left(\sum_{n=1}^{\infty} \int_{0}^{\infty} \frac{a_{n} f(x)}{x+n} d x\right)^{2}< & \frac{B\left(\lambda_{1}, \lambda_{2}\right)}{\lambda_{1}^{\lambda_{1}} \lambda_{2}^{\lambda_{2}}}\left(\int_{0}^{\infty} x^{p\left(1-\lambda_{1}\right)-1} f^{p}(x) d x\right)^{\frac{1}{p}}\left(\sum_{n=1}^{\infty} n^{q\left(1-\lambda_{2}\right)-1} a_{n}^{q}\right)^{\frac{1}{q}} \\
& \times\left(\sum_{n=1}^{\infty} \int_{0}^{\infty} \frac{x f(x) a_{n}}{(x+n)^{2}} d x\right)^{\lambda_{1}}\left(\sum_{n=1}^{\infty} \int_{0}^{\infty} \frac{n f(x) a_{n}}{(x+n)^{2}} d x\right)^{\lambda_{2}} .
\end{aligned}
$$

If we put $p=q=2, \lambda_{1}=\frac{1}{2}=\lambda_{2}$ in (2.9), we obtain the following sharper form of the halfdiscrete Hilbert inequality (1.8) ( for $\lambda=1$ )

$$
\begin{aligned}
& \left(\sum_{n=1}^{\infty} \int_{0}^{\infty} \frac{a_{n} f(x)}{x+n} d x\right)^{2} \\
& \quad<2 \pi\left(\int_{0}^{\infty} f^{2}(x) d x\right)^{\frac{1}{2}}\left(\sum_{n=1}^{\infty} a_{n}^{2}\right)^{\frac{1}{2}}\left(\sum_{n=1}^{\infty} \int_{0}^{\infty} \frac{x f(x) a_{n}}{(x+n)^{2}} d x\right)^{\frac{1}{2}}\left(\sum_{n=1}^{\infty} \int_{0}^{\infty} \frac{n f(x) a_{n}}{(x+n)^{2}} d x\right)^{\frac{1}{2}} .
\end{aligned}
$$

2. If we put $F(x, n)=\frac{a_{n} f(x)}{u(x)+\nu(n)+\mu}(\mu>0)$ in (2.1), then we have

$$
\begin{aligned}
\left(\sum_{n=n_{0}}^{\infty} \int_{b}^{c} \frac{a_{n} f(x)}{u(x)+v(n)+\mu} d x\right)^{2}< & \frac{B\left(\lambda_{1}, \lambda_{2}\right)}{\lambda_{1}^{\lambda_{1}} \lambda_{2}^{\lambda_{2}}}\left(\int_{b}^{c} \frac{[u(x)]^{p\left(1-\lambda_{1}\right)-1}}{\left[u^{\prime}(x)\right]^{p-1}} f^{p}(x) d x\right)^{\frac{1}{p}} \\
& \times\left(\sum_{n=n_{0}}^{\infty} \frac{[v(n)]^{q\left(1-\lambda_{2}\right)-1}}{\left[v^{\prime}(n)\right]^{q-1}} a_{n}^{q}\right)^{\frac{1}{q}} \\
& \times\left(\sum_{n=n_{0}}^{\infty} \int_{b}^{c} \frac{u(x) f(x) a_{n}}{(u(x)+v(n)+\mu)^{2}} d x\right)^{\lambda_{1}} \\
& \times\left(\sum_{n=n_{0}}^{\infty} \int_{b}^{c} \frac{v(n) f(x) a_{n}}{(u(x)+v(n)+\mu)^{2}} d x\right)^{\lambda_{2}},
\end{aligned}
$$

since

$$
\sum_{n=n_{0}}^{\infty} \int_{b}^{c} \frac{a_{n} f(x)}{u(x)+v(n)+\mu} d x=\sum_{n=n_{0}}^{\infty} \int_{b}^{c} \frac{u(x) f(x) a_{n}}{(u(x)+v(n)+\mu)^{2}} d x+\sum_{n=n_{0}}^{\infty} \int_{b}^{c} \frac{v(n) f(x) a_{n}}{(u(x)+v(n)+\mu)^{2}} d x
$$




$$
\begin{aligned}
& +\sum_{n=n_{0}}^{\infty} \int_{b}^{c} \frac{\mu f(x) a_{n}}{(u(x)+v(n)+\mu)^{2}} d x \\
:= & S_{1}+S_{2}+S_{3},
\end{aligned}
$$

we obtain

$$
\begin{aligned}
\sum_{n=n_{0}}^{\infty} \int_{b}^{c} \frac{a_{n} f(x)}{u(x)+v(n)+\mu} d x< & B\left(\lambda_{1}, \lambda_{2}\right)\left(\int_{b}^{c} \frac{[u(x)]^{p\left(1-\lambda_{1}\right)-1}}{\left[u^{\prime}(x)\right]^{p-1}} f^{p}(x) d x\right)^{\frac{1}{p}} \\
& \times\left(\sum_{n=n_{0}}^{\infty} \frac{[v(n)]^{q\left(1-\lambda_{2}\right)-1}}{\left[v^{\prime}(n)\right]^{q-1}} a_{n}^{q}\right)^{\frac{1}{q}} \frac{\left(\frac{S_{1}}{\lambda_{1}}\right)^{\lambda_{1}}\left(\frac{S_{2}}{\lambda_{2}}\right)^{\lambda_{2}}}{S_{1}+S_{2}+S_{3}}
\end{aligned}
$$

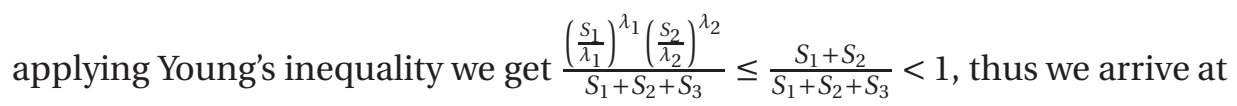

$$
\begin{aligned}
\sum_{n=n_{0}}^{\infty} \int_{b}^{c} \frac{a_{n} f(x)}{u(x)+v(n)+\mu} d x< & B\left(\lambda_{1}, \lambda_{2}\right)\left(\int_{b}^{c} \frac{[u(x)]^{p\left(1-\lambda_{1}\right)-1}}{\left[u^{\prime}(x)\right]^{p-1}} f^{p}(x) d x\right)^{\frac{1}{p}} \\
& \times\left(\sum_{n=n_{0}}^{\infty} \frac{[v(n)]^{q\left(1-\lambda_{2}\right)-1}}{\left[v^{\prime}(n)\right]^{q-1}} a_{n}^{q}\right)^{\frac{1}{q}} .
\end{aligned}
$$

3. Let $p=q=2, \lambda_{1}=\frac{1}{2}=\lambda_{2}, u(x)=x, x \in(0, \infty), v(n)=n, n \geq 1, f(x)=\frac{1}{x+1}, a_{n}=\frac{1}{n}$, then by (2.1) we obtain

$$
\begin{aligned}
& {\left[\sum_{n=1}^{\infty} \int_{0}^{\infty} F(x, n) d x\right]^{2}} \\
& \quad<2 \pi(\zeta(2))^{\frac{1}{2}}\left(\sum_{n=1}^{\infty} \int_{0}^{\infty} n x(x+1) F^{2}(x, n) d x\right)^{\frac{1}{2}}\left(\sum_{n=1}^{\infty} \int_{0}^{\infty} n^{2}(x+1) F^{2}(x, n) d x\right)^{\frac{1}{2}},
\end{aligned}
$$

where $\zeta(\alpha)$ is the Riemann zeta function. In particular if we set $F(x, n)=\frac{c_{n}}{(x+1)^{2}}, F(x, n)=\frac{g(x)}{n^{2}}$, respectively in (3.1) then we get the following Carlson's type inequalities

$$
\sum_{n=1}^{\infty} c_{n}<\frac{\pi}{6^{\frac{1}{4}}}\left\{\sum_{n=1}^{\infty} n c_{n}^{2}\right\}^{\frac{1}{4}}\left\{\sum_{n=1}^{\infty} n^{2} c_{n}^{2}\right\}^{\frac{1}{4}}
$$

and

$$
\int_{0}^{\infty} g(x) d x<\left(\frac{2 \pi \sqrt{\zeta(3)}}{\zeta(2)}\right)^{\frac{1}{2}}\left\{\int_{0}^{\infty} x(x+1) g^{2}(x) d x\right\}^{\frac{1}{4}}\left\{\int_{0}^{\infty}(x+1) g^{2}(x) d x\right\}^{\frac{1}{4}}
$$

\section{References}

[1] B. Yang, A half-discrete Hilbert's inequality, J Guangdong Univ Edu., 31(3) (2011), 1-7.

[2] B. Yang and Q. Chen, A half-discrete Hilbert-type inequality with a homogeneous kernel and an extension, Journal of Inequalities and Applications, 2011 2011:124. 
[3] G. H. Hardy, J. E. Littlewood and G. Polya, Inequalities, Cambridge Univ. Press, London, 1952.

[4] L. E. Azar, A relation between Hilbert and Carlson inequalities, Journal of Inequalities and Applications, 2012, 2012:277.

[5] L. Larsson, L. Maligranda, J. Pecarić and L. E. Persson, Multiplicative Inequalities of Carlson Type and Interpolation,World Scientific Publication co. Pty. Hackensack, NJ, 2006.

Department of Mathematics, Al al-Bayt University, P.O. Box: 130095 Mafraq, Jordan.

E-mail: azar1@aabu.edu.jo 